\title{
Prevalencia de maloclusiones en pacientes del servicio de ortodoncia de la Facultad de Odontología UNNE 2013
}

Prevalencia of maloclusiones in patients to the service of Orthodontics of the Faculty of

Odontolgy UNNE 2013
Prevalência de má oclusão em pacientes do serviço de ortodontia da Faculdade de

Odontologia UNNE 2013
Andrea Ruiz Díaz Becaria de Grado Ciencia y Técnica.

\section{I. Collante de Benitez}

Prof. Cátedra Fundamentos de la Ortodoncia.

\section{A. Guiglione}

Prof. Cátedra Introducción a la Odontología.

L. Palcikwoski Becaria posgrado Ciencia y Técnica.

\section{Resumen}

Estudios epidemiológicos han demostrado que la maloclusión se presenta con tasas de prevalencia altas, debido a que se manifiesta en más del $60 \%$ de la población. El objetivo de este trabajo fue determinar clínicamente la clase de Angle, en los pacientes que concurrieron al Servicio de Ortodoncia de la Facultad de Odontología UNNE, durante el primer semestre del año 2013. Materiales y métodos: es un estudio epidemiológico transversal. La muestra estuvo constituida por un total de 70 pacientes, comprendido entre los 7 y 25 años de edad. Se tuvo en cuenta los siguientes criterios de inclusión: pacientes con los cuatros primeros molares permanentes erupcionados, $y$ sin tratamientos previos de aparatología funcional o fija. Los criterios de exclusión fueron: pacientes con restauraciones deficientes, $y$ pacientes portadores de prótesis. Para la evaluación clínica se utilizo espejo bucal, separadores o espandex, pinza y rollos de algodón. Se documentaron los casos en modelos de yeso y fotografías digitales. Resultados: los resultados obtenidos fueron los siguientes 27 pacientes $(34,28 \%)$ fueron portadores de Clase I, 23 pacientes $(32,85 \%)$ resultaron portadores de Clase II y los 20 restantes (28,57\%) fueron portadores de clase III. Conclusión: estos resultados hallados son similares a estadísticas mundiales. 
REVISTA FACULTAD DE ODONTOLOGÍA

ISSN No 1668-7280 - Vol. VIII No 1 - 2015

22
INVESTIGACIÓN

Ruiz Díaz - Collante de Benitez - Guiglione - Palcikwoski
Palabras claves

Anomalías, clasificación de Angle, clasificación de maloclusiones.

\section{Abstract}

Epidemiological studies have demonstrated that the malocclusion appers with rates of prevalencia discharges, since it develops in more than $60 \%$ of the population. The aim of this work was to determine clinical Angle's class, in the patients who meet to the Service dental Faculty of dental UNNE. during the first half of the year 2013. The materials and methods: it is an epidemiological transverse study. The sample was constituted by a total of 70 patients, between the 7 and 25 years of age. The following inclusion criteria were taken into account: the four patients with first erupted molars without pretreatment or fixed functional appliances. The criteria of exclusion were: patients with deficient restorations, and patient carriers of prothesis. For the clinical evaluation I use set of mouth mirror, dividers or espandex, tweezer and cotton rolls. The documentation of the cases was in models of plaster and digital photographies. Results: the obtained results were 27 patients (34.28\%) were carriers of Class I, 23 patients $(32.85 \%)$ were carriers of Clase II and the 20 remaining ones $(28.57 \%)$ were carriers of Class III. Conclusion: these found results are similar to world statistics.

\section{Key words}

Anomalies, Angle's classification, Classification of maloclusiones.

\section{Resumo}

Estudos epidemiológicos têm mostrado que a má oclusão ocorre com taxas de prevalência elevadas, desde que ele se desenvolve em mais de $60 \%$ da população. Terapéutico de forma diferente. $O$ objetivo deste estudo foi clinicamente determinar o tipo de ângulo, nos pacientes que freqüentam o serviço de Ortodontia da faculdade de Odontologia de UNNE, durante o primeiro semester do ano 2013. Materiais e métodos: um estudo epidemiológico transversal. A amostra consistiu de um total de $\mathbf{7 0}$ pacientes, entre os 7 e 25 anos de idade. Tendo em conta os seguintes critérios de inclusão: pacientes com os quatro primeiros molares erupção e sem tratamentos anteriores de aparelhos funcionais ou fixos. Critérios de exclusão foram: pacientes com restaurações deficientes e doentes com uma prótese. Para a avaliação clínica, eu uso espelho de boca, separadores ou elastano, algodão e braçadeira rolos. Então foi a documentação dos casos em modelos de gesso e fotografias digitais. Resultados: os resultados foram os seguintes de 70 pacientes que formaram a amostra todos os 27 pacientes $(34.28 \%)$ eram portadores de classe I, 23 pacientes (32.85\%) e os restantes 20 (28.57\%) eram portadores de classe III. Conclusão: estes resultados encontrados são semelhantes às estatísticas globais.

\section{Palavras chave}

Anomalia, Classificação de Angle, Classificação da má oclusão.

\section{Introducción}

Estudios epidemiológicos hacen referencia a que la maloclusión se presenta con tasas de prevalencia considerablemente altas, exhibiendo variaciones entre diferentes países, etnias y grupo etarios; observándose una prevalencia más alta en las sociedades urbanas, lo cual ha llevado a que se considere como una enfermedad de la civilización'.

En cuanto a su distribución, de acuerdo al tipo de maloclusión, en la actualidad diferentes estudios realizados, han demostrado que, la clase I (60\%) es hasta cinco veces más frecuente en comparación con la clase II (27\%) y III (5\%). No obstante, aún cuando la clase I es de las tres categorías de maloclusión la de menor severidad, existen cinco variaciones en su manifestación que orientan su abordaje diagnostico y terapéutico de diferente manera ${ }^{1,2,3,4}$.

Las maloclusiones instituyen un problema de salud pública desde el punto de vista de la odontología, precediendo a la caries dental ${ }^{5}$.

La oclusión puede ser alterada por distintos factores genéticos $y$ adquiridos que repercuten durante el proceso evolutivo de las denticiones mixtas y permanentes, también es de destacar 
REVISTA FACULTAD DE ODONTOLOGÍA

ISSN No 1668-7280 - Vol. VIII № 1 - 2015

23
INVESTIGACIÓN

Prevalencia de maloclusiones en pacientes del servicio de ortodoncia de la Facultad de Odontología UNNE 2013 como otro factor la herencia. En ocasiones es de carácter multifactorial, interactuando e inclusive sobreponiéndose una causa sobre otra lo que dificulta su diagnostico ${ }^{5,6}$.

Para Proffit W, la maloclusión no es más que una alteración del crecimiento. En la mayoría de los casos, la maloclusion no se debe a procesos patológicos, si no a una moderada distorsión del desarrollo normal. En ocasiones, es posible demostrar la existencia de una causa específica aislada, como la maloclusión característica que aparece en algunos síndromes genéticos ${ }^{7}$.

Para poder estudiar la maloclusión se utiliza la clasificación de Angle, quien la describió a finales del siglo IXX, es incompleta, porque solo atiende al problema oclusal sagitalmente, no tiene en cuenta las relaciones transversales ni verticales, como así tampoco las anomalías de las bases óseas. Sin embargo es mundialmente, la primera clasificación ortodóncica que continua siendo útil, y reconocida por sus estudios cefalométricos que se realizan y por programas informáticos que existen en el mercado $7,8,9$.

Angle basa su clasificación en las relaciones de oclusión mesio-distales de los primeros molares permanentes, relación a la que llamo llave o clave de la oclusión, cuando la cúspide mesio-vesribular del primer molar superior permanente ocluye con el surco mesio-vestibular del primer molar inferior permanente; sosteniendo como principio, la normal implantación de los primeros molares superiores en el maxilar, siendo los primeros molares inferiores y maxilar inferior los susceptibles a cambiar de posición ${ }^{8,10}$.

Contempla tres categorías, designadas con cifras romanas: la maloclusión Clase I, II y III. A las clasificaciones Clase II y III las divide y subdivide en subgrupos reconocibles; englobando en una sola categoría a la Clase I, a pesar de ser la alteración en la oclusión dental con mayor prevalencia, lo que genera un esfuerzo para el profesional odontólogo para clarificar los conceptos con respecto a las discrepancias más comúnmente vistas en las maloclusiones, la cual es causada, en la mayoría de las veces por displasias dentarias y con menor frecuencia por displasias esqueléticas u óseas"

Clase I: esta clase está determinada por la relación mesio-distal normal de los primeros molares permanetes. Se cumplen las claves de la oclusión en ambos lados. Los músculos de los labios y lengua funcionan normalmente, con más probabilidad que en la Clase II y III. Los dientes anteriores pueden presentar distintas anomalías de posición; entre ellas las más frecuentes son: I) retrusiones superiores e inferiores con apiñamiento dentario y por lo general canino fuera de la arcada. Presenta falta de desarrollo antero posterior y transversal de los maxilares. 2) retrusión superior únicamente, con maxilar inferior normal; canino superior retenido o en vestíbulo-intraversión, sin o con poco espacio, es la aparente Clase III, por su aspecto facial y relación bilabial. 3) protrusión superior, dientes en vestibuloversión y con diastema, por su aspecto facial y relación bilabial aparente Clase II. 4) protrusión inferior. 5) protrusión superior e inferior.

Clase II: corresponde a la relación distal de los primeros molares inferiores y de la arcada en consecuencia. Dentro de esta clase, se establece dos divisiones, y en cada una de ellas una subdivisión, que las designa con números arábigos.

Clase II: $I^{\circ}$ división: caracterizada por la vestíbulo-versión de los incisivos superiores, el arco superior estrecho, labio superior hipotónicos y separado del inferior, entre los que asoman los dientes superiores. Las piezas inferiores en extraversión alcanzan la mucosa palatina. Musculatura blanda y lábil. Asociada a la respiración bucal.

Angle dispone una subdivisión para los casos de unilateralidad en la relación distal, es decir, de un lado relación distal y en la opuesta relación molar.

Clase II: $2^{\circ}$ división: definida por la linguo - versión de los incisivos. Puede afectar la linguoversión a los dos incisivos centrales y los laterales aparentar una vestíbulo-versión; o bien afectar a los cuatros incisivos, y en este caso son los caninos los vestibulizados. Los superiores ocultan casi completamente a los inferiores llegando en determinados casos a tocar la encía vestibular inferior, mientras que los inferiores alcanzan la encía palatina, en los casos más pronunciados. Son respiradores normales y presentan labios normales o aumentados y surco labio nasal y labio mentoniano pronunciados. Su musculatura presenta gran tonicidad. 
La subdivisión corresponde a la relación distal de los molares unilateral, es decir la clave de la oclusión molar está rotada por la relación en un lado mientras que el opuesto está conservada o normal.

Clase III: está determinada por la relación mesial de los molares inferiores y de la arcada en consecuencia, más de la mitad del ancho de una cúspide. Los incisivos inferiores por delante de los superiores, y tapando parcial o totalmente a los superiores. En algunos casos por deformación mandibular están por delante pero separados por una distancia que puede llegar hasta más de un centímetro.

Angle establece la subdivisión cuando de un lado la clave está rotada en sentido mesial y en el opuesto está conservado (7)(11).

Con base en estos antecedentes, el presente estudio, permitirá a los odontólogos enriquecer el panorama de estas alteraciones, así como establecer parámetros y criterio clínico en los casos de maloclusiones en los pacientes que concurren al servicio de Ortodoncia de la Facultad de Odontología U.N.N.E.

\section{Materiales y Métodos}

Como objetivo se estableció: Determinar clínicamente la clase de Angle en pacientes que concurren al Servicio de Ortodoncia de la Facultad de Odontología UNNE, durante el año 20I3.

Para ello, se realizó el estudio de un total de 70 pacientes que concurrieron al Servicio de
Ortodoncia, y cumplieron con los criterios de inclusión.

Los cuales fueron:

- Pacientes con los cuatros primeros molares erupcionados.

- Pacientes sin tratamientos previos con aparatología funcional o fija

Los criterios de exclusión fueron los siguientes:

- Pacientes con restauraciones deficientes

- Pacientes portadores de prótesis

Se utilizó instrumental descartable de exploración, consistente en espejo bucal, separadores o espandex, pinza y rollos de algodón. Luego se realizó la documentación de los casos en modelos de yeso y fotografías digitales.

\section{Resultados}

De los 200 pacientes que concurrieron al Servicio de Ortodoncia se seleccionaron 70 que cumplieron con los requisitos de inclusión. La edad de los pacientes osciló entre 7 y 25 años de edad.

Se pudo constatar que la prevalencia de de clase I fue de $34,28 \%$, debido a que 27 pacientes del total de la muestra seleccionada la presentaron. La clase II se presentó en 23 pacientes, siendo su prevalencia del $32,85 \%$. En cuanto que el 28,57 $\%$ del total de la muestra, 20 pacientes fueron portadores de clase III (Fig. I).

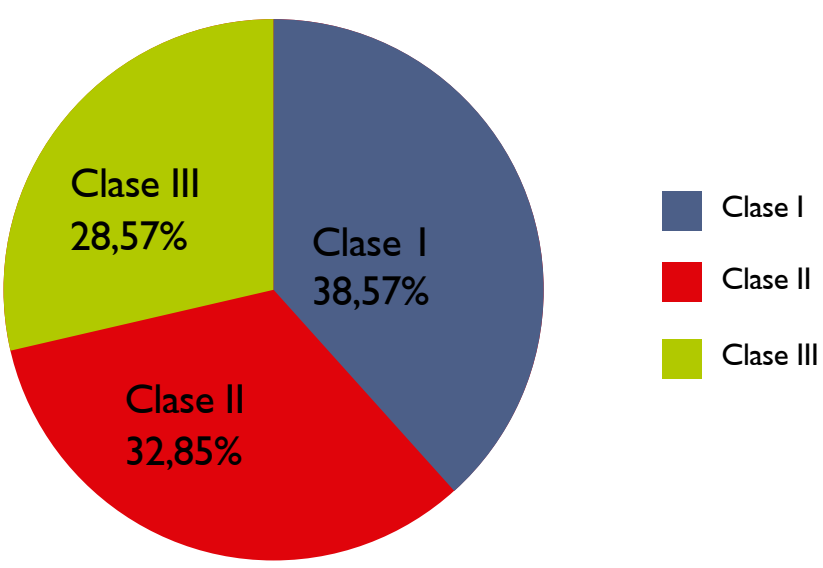

Figura I. Prevalencia de maloclusiones. 


\section{Discusión}

En este estudió, es de considerar que la muestra estuvo integrada por pacientes que concurrieron a un servicio de ortodoncia, derivados por otros profesionales o bien en forma espontánea con algún signo de maloclusión muy evidente lo cual se debe tener en cuenta al apreciar los datos obtenidos.

Se puede apreciar que el estudio realizado Murrieta Pruneda y colaboradores (I), la taza de prevalencia de maloclusión del total de la muestra es de $96,40 \%$, de ello la clase I es la más frecuente representando el $72,80 \%$ de los casos de maloclusión detectado, al igual que el estudio de Reddy y colaboradores ${ }^{(3)}$ en que la prevalencia de Clase I es de 78,60\%. En ambos casos le siguen en orden la clase II y en ultimo término la clase III. Al comparar los resultados de los estudios ante citados con el presente, los porcentajes no son similares, sin embargo el orden de prevalencia es el mismo.

En la publicación de Urrego Burbano y colaboradores ${ }^{(4)}$, el orden continúa siendo el mismo.

En el estudio de Sidiauskas y Lopatiene (2) el orden de prevalencia sigue siendo el mismo, y la prevalencia de clase II, $27,70 \%$ es muy próxima al del presente estudio donde el valor alcanza a $32,85 \%$.

\section{Conclusión}

Se puede concluir que la clase I, es la maloclusión más prevalerte, a la que le sigue la clase II y en último lugar la clase III.

\section{Bibliografía}

I. Murrieta Pruneda JF, Cruz Díaz PA y colaboradores. Prevalencia de Maloclusiones Dentales en un Grupo de Adolescente Mexicanos y su Relación con la Edad y el Género. Acta Venezolana 2007; 45:74-78. En: http://www.actaodontologi-

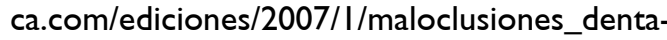
les.asp

3. Sidiauskas A y Lopatiene K. The prevalence of malocclusion among 7-15- year-old Lithuanian schoolchildren. Medicina (Kaunas). 2009; 45(2): 147-52.

4. Reddy ER, Manjula M y colaboradores. Prevalence of maloclusion among 6 to 10 yer old Nalgonda School Children. J int Oral Health. 2013 Dec; 5(6):49-54. Epub 20I3Dec 26.

5. Urrego - Burbano PA, Jiménez -Arroyave LP y colaboradores. Perfil epidemiológico de la oclusión dental en escolares de Envigado, Colombia. Rev Salud Pública (Bogota). 20II Dec; 13 (6): $|0| 0-2 \mid$.

6. Acevedo K, Belussi K y Otros. Estudio descripto de prevalencia de las causas de Maloclusiones en el Servicio de Odontología del Hospital San Juan de Dios". Revista Latinoamericana de Ortodoncia y Odontopediatría. 2013

7. Graber TM, Vanardall RL. Ortodoncia Principios Generales y Técnicas. 3ra ed. Bues Aires: Panamericana, 1997. pl8-24

8. Proffit WR y colaboradores. Ortodoncia Contemporánea. 4ta ed. España: Elsevier Mosby. 2008, p.218-220.

9. Rojas G y Otros. Tipo de Maloclusiones Dentales más frecuentes en los pacientes del Diplomado de Ortodoncia Interceptiva de la Universidad Gran Marriscal de Ayacucho 2007 - 2008. Latinoamericana de Ortodoncia y Odontopediatría 2010

10. Gregoret J, Tuber E. Ortodoncia y Cirugía Ortognática Diagnostico y planificación. 2da ed. Madrid España: NM ediciones. 2008. P I0I-I I4.

II. Ustrell Torrent JM y Duran von Arx J. Ortodoncia. 2da ed. Barcelona: Edicions de la Universitat de Barcelona. 2002, p. 109-124

12. Cannut Brusola JA. Ortodoncia Clínica y Terapéutica. 2da ed. Masson S.A: Venezuela. 2000. p 465-492, 535-55I, 573-580, 599-607. 